\title{
Unusual Presentation of Non-Hodgkin's B-Cell Lymphoma with Unilateral Right Limb Lymphedema
}

\author{
Massimo Bolognesi ${ }^{*}$, Diletta Bolognesi² \\ ${ }^{1}$ General Practice Medicine-Primary Care, Cesena, Italy \\ ${ }^{2}$ Territorial Medicine-Primary Care, Cesena, Italy \\ Email: ${ }^{*}$ massbolo1@tin.it
}

Received 9 September 2014; revised 5 October 2014; accepted 3 November 2014

Copyright (C) 2014 by authors and Scientific Research Publishing Inc.

This work is licensed under the Creative Commons Attribution International License (CC BY).

http://creativecommons.org/licenses/by/4.0/

c) (i)

\begin{abstract}
Background: In clinical practice and setting of general practice it is common to see patients with leg edema. To correctly identify the etiology of the edema and then properly manage the cause is not always easy. The unilateral lymphedema of the lower limb has rarely been reported as an initial presentation for lymphoma, especially in females, usually without classic signs or symptoms, but often with inguinal lymphadenopathy or abdominal masses. Case Report: In this article, we report a rare case of unilateral lower limb edema in a healthy obese woman who complained about the appearance of the disease for several months and for whom deep vein thrombosis and other diseases had been excluded. The histological examination of the biopsy of an enlarged lymph node in the right groin, which was compressing the iliac and femoral vein, revealed the presence of B cell non-Hodgkin lymphoma with high-grade malignancy. Conclusions: A common challenge for primary care physicians is to determine the cause and find an effective treatment for leg edema of unclear etiology. Non-Hodgkin's B-cell Lymphoma should be considered in the differential diagnosis in patients with unilateral leg edema when the swelling is chronic and deep venous thrombosis is promptly excluded.
\end{abstract}

\section{Keywords}

Component, Unilateral Leg Edema, Non-Hodgkin's B-Cell Lymphoma, Inguinal Lymphadenopathy

\section{Introduction}

A common challenge for primary care physicians is to determine the cause and find an effective treatment for

"Corresponding author.

How to cite this paper: Bolognesi, M. and Bolognesi, D. (2014) Unusual Presentation of Non-Hodgkin's B-Cell Lymphoma with Unilateral Right Limb Lymphedema. Case Reports in Clinical Medicine, 3, 577-582.

http://dx.doi.org/10.4236/crcm.2014.311125 
leg edema of unclear etiology. The most likely cause of leg edema in patients over 50 years of age is venous insufficiency. There are two types of leg edema: venous edema and lymphedema. Venous edema consists of excess low-viscosity, protein-poor interstitial fluid resulting from increased capillary filtration that cannot be accommodated by a normal lymphatic system [1].

Lymphedema consists of excess protein-rich interstitial fluid within the skin and subcutaneous tissue resulting from lymphatic dysfunction [2].

Primary lymphedema is a rare disorder that is divided into 3 types according to age of presentation [3].

Unilateral leg edema is a frequent clinical problem, usually reflecting underlying vascular disease. Unilateral leg edema is generally due to a local cause such as deep vein thrombosis, venous insufficiency, or lymphedema. Bilateral edema can be due to a local cause or systemic disease, such as heart failure or kidney disease. Generalized edema is due to systemic disease [4].

Secondary lymphedema is much more common than primary, and the cause is generally apparent from the history [5]. The most common causes of leg lymphedema are tumors (e.g., lymphoma, prostate cancer, ovarian cancer), surgery involving lymphatics, radiation therapy, and infection (bacterial infection or filariasis) [6].

To our knowledge there has been only one previous report of a case of unilateral lymphoma-related leg edema [7] in the English-language literature. An original study [8] retrospectively found a 1.1 percent prevalence of malignancy in primary care patients presenting themselves to the office with unexplained lymphadenopathy.

This report describes an uncommon cause of obstructive lymphedema due to enlarged lumbar aortic, iliac and inguinal lymphadenopathy of malignant lymphoma, in a 70-year-old obese female in the setting of general practice. For this patient, the diagnosis of lymphoma was overlooked during the initial consultation due to lack of symptoms and signs of systemic disease.

\section{Case Report}

A 70-year-old female suffering from obesity and high blood pressure presented herself to our office complaining of pain with swelling of the right leg which had started some months before. She denied any weakness, fever, night sweats, weight loss, dyspnea, exercise intolerance, cough and headache. She reported no prolonged immobilization or bed rest. Cardiac examination was normal and the chest was clinically clear.

No hepatosplenomegaly was found but enlarged right inguinal lymph nodes were observed. No other lymphadenopathies were palpable in the neck and axilla.

Physical examination of the lower extremities revealed severe right leg swelling with pitting edema, erythematous skin, increased warmth and pain on pressure of the calf. The right leg appeared grossly swollen as compared to the left lower limb. Femoral and popliteal arterial pulses were not palpable because of edema (See Figure 1).

The chest X-ray and the two-dimensional transthoracic echocardiography exam were normal.
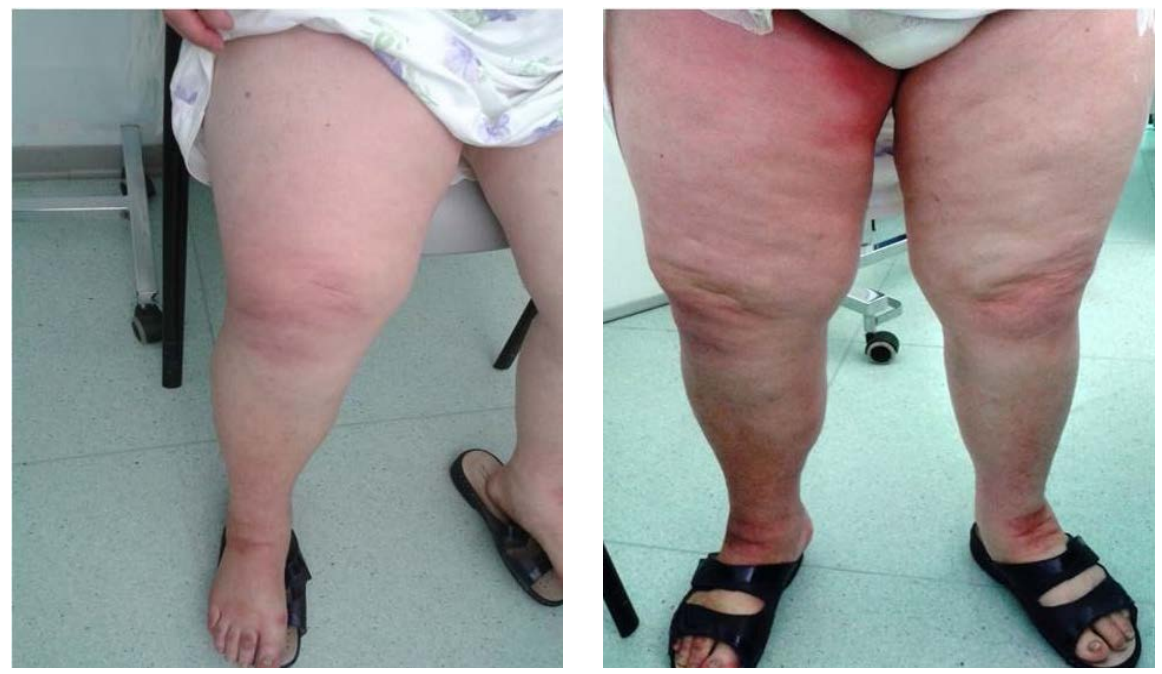

Figure 1. Unilateral limb edema. 
Laboratory evaluation was unremarkable except for the presence of mildly elevated inflammatory markers with a slight increase in d-dimer ( $>700 \mathrm{mg} / \mathrm{dl}$ ). Subsequently a Doppler ultrasonography of the lower extremities was performed but no sign of deep vein thrombosis was detected. Therefore the patient underwent to an abdomen ultrasound scan that showed the presence of a complex right inguinal lymphadenopathy compressing the ipsilateral femoral and iliac vein. Also the abdomen CT scan showed enlarged right lumbar para-aortic lymph nodes extended to iliac-obturator stations as well as the ipsilateral inguinal-femoral lymph nodes, as seen by ultrasonography (See Figure 2).

It was concluded that the mechanical obstruction of the blood vessels in the groin determined the lower limb lymphedema. Consequently, the patient was admitted to hospital to carry out a biopsy of an enlarged inguinal lymph node for histological definition of suspected lymphoproliferative disease. In fact biopsy showed complete effacement of nodal architecture by malignant tumor, comprising diffused cells having large pleomorphic vesicular nuclei with prominent nucleoli, suggesting the existence of diffused B-cell lymphoblastic non-Hodgkin's lymphoma. Consequently the patient was referred to the oncology department and underwent chemotherapy with improvement of the right limb lymphedema (See Figure 3).
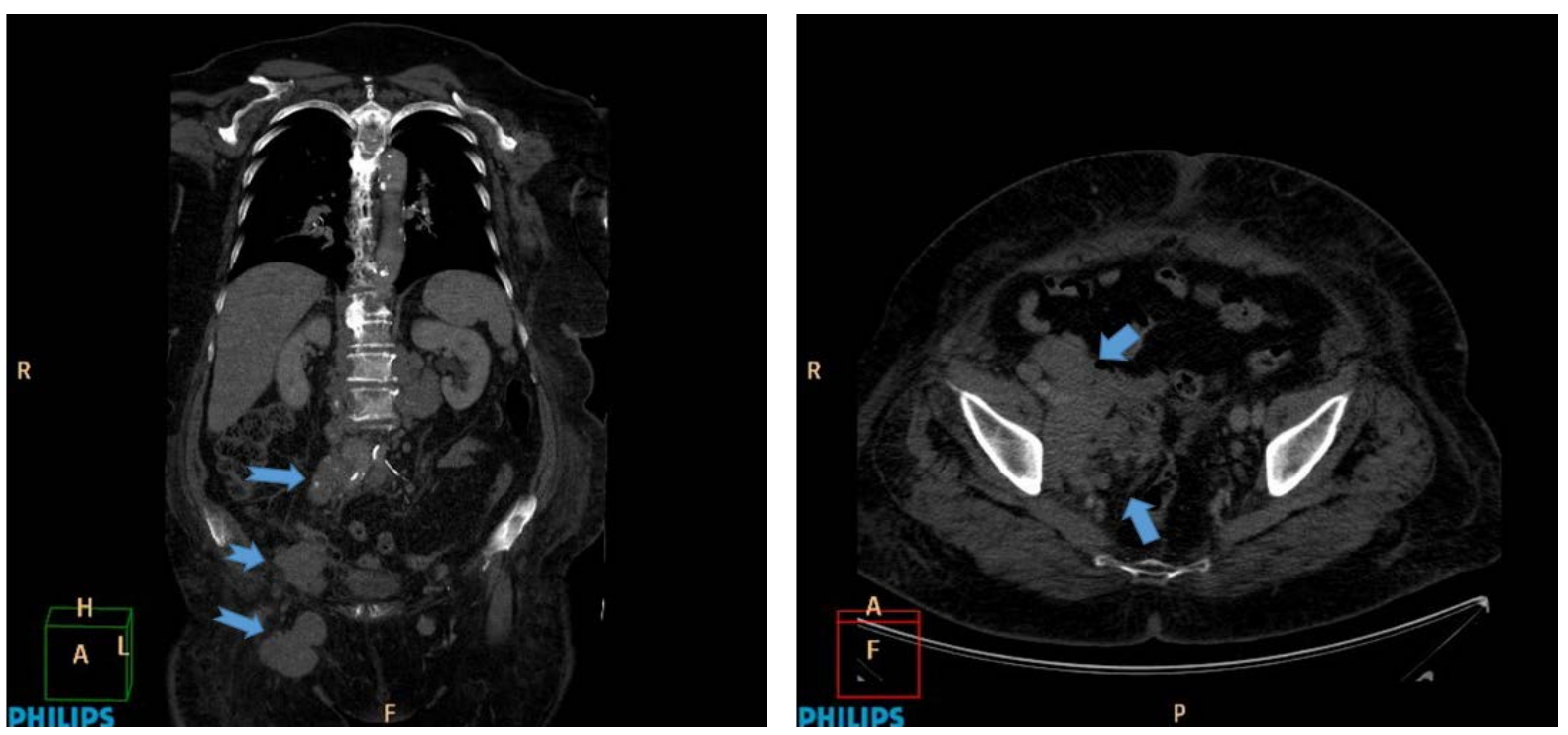

Figure 2. Abdomen CT scan showing enlarged right lumbar para-aortic, iliac and inguinal lymph nodes (see arrows).

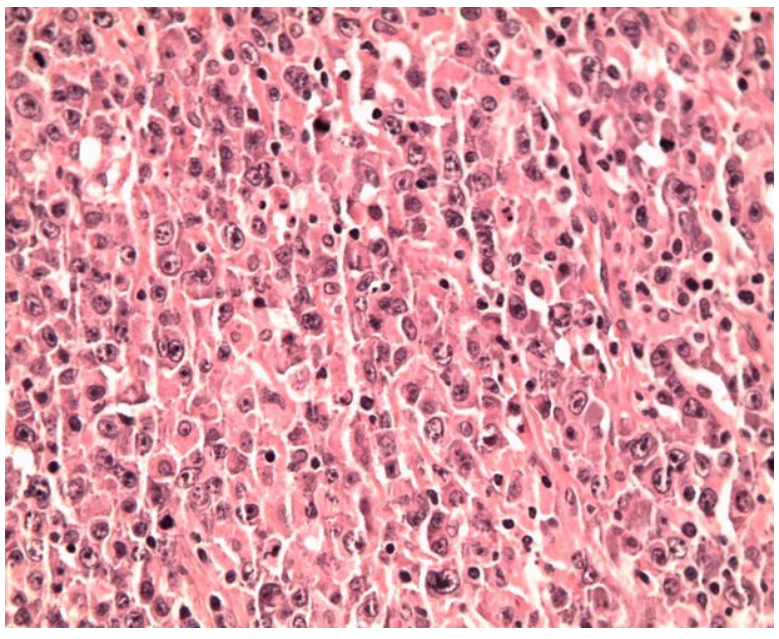

(a)

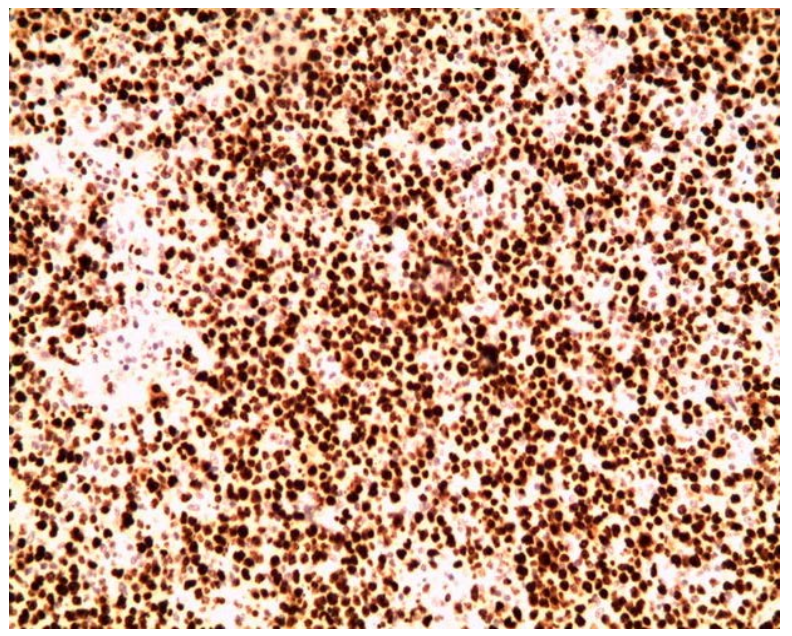

(b)

Figure 3. Histopathology findings-Panel A shows intense positivity of lymphoid elements with CD-20, a marker of B lymphocytes; Panel B shows intense diffuse positivity for Ki-67 as expression of high proliferative index. 


\section{Discussion}

Lymph node enlargement may occur because of proliferation of cells of the lymphocyte and monocyte-macrophage systems, usually in response to antigenic stimulus, or may be due to infiltration by inflammatory cells in infections involving lymph nodes (lymphadenitis).

In situ proliferation of malignant lymphocytes or macrophages, infiltration of nodes by metastatic malignant cells or infiltration of lymph nodes by metabolite laden macrophages are the storage of diseases [9].

When the cause for the lymphadenopathy remains unexplained, the physician must decide whether to pursue a specific diagnosis. The decision will depend primarily on the clinical setting as determined by the patient's age, the duration of the lymphadenopathy and the characteristics and location of the nodes.

Using the factors above as guidance, a thorough history and physical examination should allow physicians to categorize individual cases of lymphadenopathy according to the algorithm as posted by Ferrer [10].

In developed countries, the most common cause of secondary lymphoedema is malignancy.

On the other hand, filariasisis considered the most common cause of secondary lymphoedema in developing countries [11].

The appearance of pronounced lymphedema of a lower extremity due an obstruction of iliac and femoral vein in the groin, as the first clinical manifestation and presenting sign of Hodgkin's disease is extremely rare [12] [13].

The development of lymphedema is a slow, insidious process starting in the most distal portion of the extremity.

Ninety-five percent of unilateral lymphedema involves obstruction of lymphatics due to infection, postoperative lymphatic disruption and malignancy. Carcinoma of prostate in males and lymphomas and pelvic tumours in females are commonly responsible. Presentation of lymphoma, as leg edema, is uncommon, especially in the absence of generalized lymphadenopathy.

Diffuse large cell lymphomas are the most common types of NHL and account for $60 \%-70 \%$ of aggressive lymphoid neoplasm and $85 \%$ of these originate in B-cells. The mean age of presentation is $65-70$ years of age and patients present rapid symptomatic lymphadenopathy. B-symptoms (fever, night sweats, weight loss $>10 \%$ of normal body weight), skin rash and symptoms pertinent to extra-nodal involvement were absent [14].

Notoriously non-Hodgkin's lymphoma typically presents with painless progressive lymphadenopathy and commonly with bilateral leg edema [15].

Unilateral lower extremity lymphedema is considered a possible but rare initial presentation for non-Hodgkin's lymphoma [16].

This case report confirms that this is more common in women [17] and there are usually no associated B-symptoms (e.g., weight loss, anorexia and night sweats), making for low clinical suspicion in our female patient. A close examination for inguinal lymphadenopathy or abdominal masses may provide a key clue for the diagnosis of lymphoma.

It follows that to make an early diagnosis of non-Hodgkin's lymphoma subtype an excisional biopsy with histological examination of the lymph nodes is mandatory [18].

Although the advent of new immunohistochemical analytic techniques has increased the sensitivity and specificity of fine-needle aspiration [19] excisional biopsy remains the gold standard diagnostic procedure.

\section{Conclusions}

In conclusion, the aim of this case is to draw physicians attention as they should not underestimate the unusual presentation of lymphedema in patients over 50 years old, given the need to perform an early diagnosis and specific therapy for lymphoma.

This entity should be considered in the differential diagnosis in patients with unilateral leg edema when the swelling is chronic and deep venous thrombosis is promptly excluded.

\section{Acknowledgements}

The authors thank Dr. M. Ferretti and G. Caruso for the courtesy to of providing images of the clinical case.

\section{Manuscript Type}

Case report. 
Both authors contributed to conception and design, manuscript preparation, read and approved the final manuscript.

Both authors abide by the Association for Medical Ethics (AME) ethical rules of disclosure.

\section{Competing Interests}

None declared.

\section{Conflict of Interests}

None declared.

\section{Consent}

Written informed consent was obtained from the patient for publication of this case study and the accompanying images are for scientific purposes.

\section{References}

[1] Gorman, W.P., Davis, K.R. and Donnelly, R. (2000) ABC of Arterial and Venous Disease. Swollen Lower Limb-1: General Assessment and Deep Vein Thrombosis. BMJ, 320, 1453-1456. http://dx.doi.org/10.1136/bmj.320.7247.1453

[2] Mortimer, P.S. (2000) Swollen Lower Limb-2: Lymphoedema. BMJ, 320, 1527-1529. http://dx.doi.org/10.1136/bmj.320.7248.1527

[3] Szuba, A. and Rockson, S.G. (1998) Lymphedema: Classification, Diagnosis, and Therapy. Vascular Medicine, 3, 145146. http://dx.doi.org/10.1177/1358836X9800300209

[4] Yale, S.H. and Mazza, J.J. (2001) Approach to Diagnosing Lower Extremity Edema. Comprehensive Therapy, 27, 242252. http://dx.doi.org/10.1007/s12019-001-0021-5

[5] Smith, R.D., Spittel, J.A. and Schirger, A. (1963) Secondary Lymphedema of the Leg: Its Characteristics and Diagnostic Implications. JAMA, 185, 116-118 http://dx.doi.org/10.1001/jama.1963.03060020040018

[6] Kinmonth, J.B., Taylor, G.W., Tracy, G.D. and Marsh, J.D. (1957) Primary Lymph-Oedema. Clinical and Lymphangiographic Studies of a Series of 107 Patients in Which the Lower Limbs Were Affected. British Journal of Surgery, 45, $1 \pm 10$.

[7] Akritidis, N. and Baxevanos, G. (2007) Unilateral Lymphoma-Related Leg Edema. Journal of Clinical Oncology, 25, 5523-5524

[8] Fijten, G.H. and Blijham, G.H. (1988) Unexplained Lymphadenopathy in Family Practice. An Evaluation of the Probability of Malignant Causes and the Effectiveness of Physicians’ Workup. The Journal of Family Practice, 27, 373376.

[9] Harry, L. and Loachim, J.B. (1994) Lymph Node Pathology. 2nd Edition, Olippincott Company, Philadelphia.

[10] Ferrer, R. (1998) Lymphadenopathy: Differential Diagnosis and Evaluation. American Family Physician, 58, 1315.

[11] Tiwari, A., Cheng, K.S., Button, M., Myint, F. and Hamilton, G. (2003) Differential Diagnosis, Investigation, and Current Treatment of Lower Limb Lymphedema. JAMA Surgery, 138, 152-161. http://dx.doi.org/10.1001/archsurg.138.2.152

[12] Mauch, P.M., Kalish, L.A., Kadin, M., Coleman, C.N., Osteen, R. and Hellman, S. (1993) Patterns of Presentation of Hodgkin Disease. Implications for Etiology and Pathogenesis. Cancer, 71, 2062-2071. http://dx.doi.org/10.1002/1097-0142(19930315)71:6<2062::AID-CNCR2820710622>3.0.CO;2-0

[13] Rigas, A., Kostakis, A., Safioleas, M., Vaos, G., Zonas, C. and Skalkeas, G. (1981) Lymphedema as the First Manifestation of Hodgkin's Disease. Lymphology, 14, 138-140.

[14] Halilbasic, A., Mesic, E., Cikusic, E. and Arnautovic, A. (2006) Non-Hodgkin’s Lymphoma in the North-East Bosnia: Changes in Biological Aggressiveness and Primary Presentation of the Disease. Medicinski Arhiv (Medical Archives), 60, 78-83.

[15] Shankland, K.R., Armitage, J.O. and Hancock, B.W. (2012) Non-Hodgkin Lymphoma. Lancet, 380, 848-857. http://dx.doi.org/10.1016/S0140-6736(12)60605-9

[16] Sajid, T., Intisar-ul-Haq, Haq, I. and Chaudhary, A.K. (2009) An Obscure Cause of Leg Edema, Non-Hodgkin’s Lymphoma. Journal of the College of Physicians and Surgeons-Pakistan, 19, 254-256.

[17] Hawkins, K.A., Amorosi, E.L. and Silber, R. (1980) Unilateral Leg Edema. A Symptom of Lymphoma. JAMA, 244, 
2640-2641. http://dx.doi.org/10.1001/jama.1980.03310230042023

[18] Armitage, J.O. (2007) How I Treat Patients with Diffuse Large B-Cell Lymphoma. Blood, 110, 29-36. http://dx.doi.org/10.1182/blood-2007-01-041871

[19] Dunphy, C.H. and Ramos, R. (1997) Combining Fine-Needle Aspiration and Flow Cytometric Immunophenotyping in Evaluation of Nodal and Extranodal Sites for Possible Lymphoma: A Retrospective Review. Diagnostic Cytopathology, 16, 200-206. 
Scientific Research Publishing (SCIRP) is one of the largest Open Access journal publishers. It is currently publishing more than 200 open access, online, peer-reviewed journals covering a wide range of academic disciplines. SCIRP serves the worldwide academic communities and contributes to the progress and application of science with its publication.

Other selected journals from SCIRP are listed as below. Submit your manuscript to us via either submit@scirp.org or Online Submission Portal.
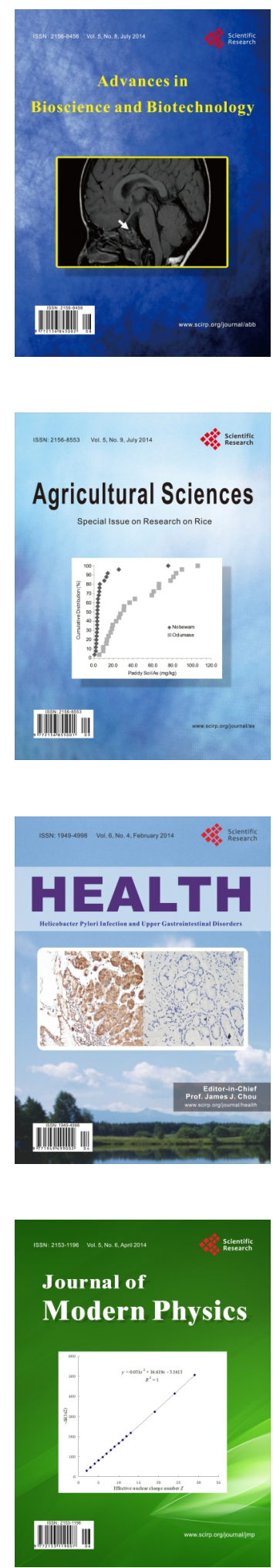
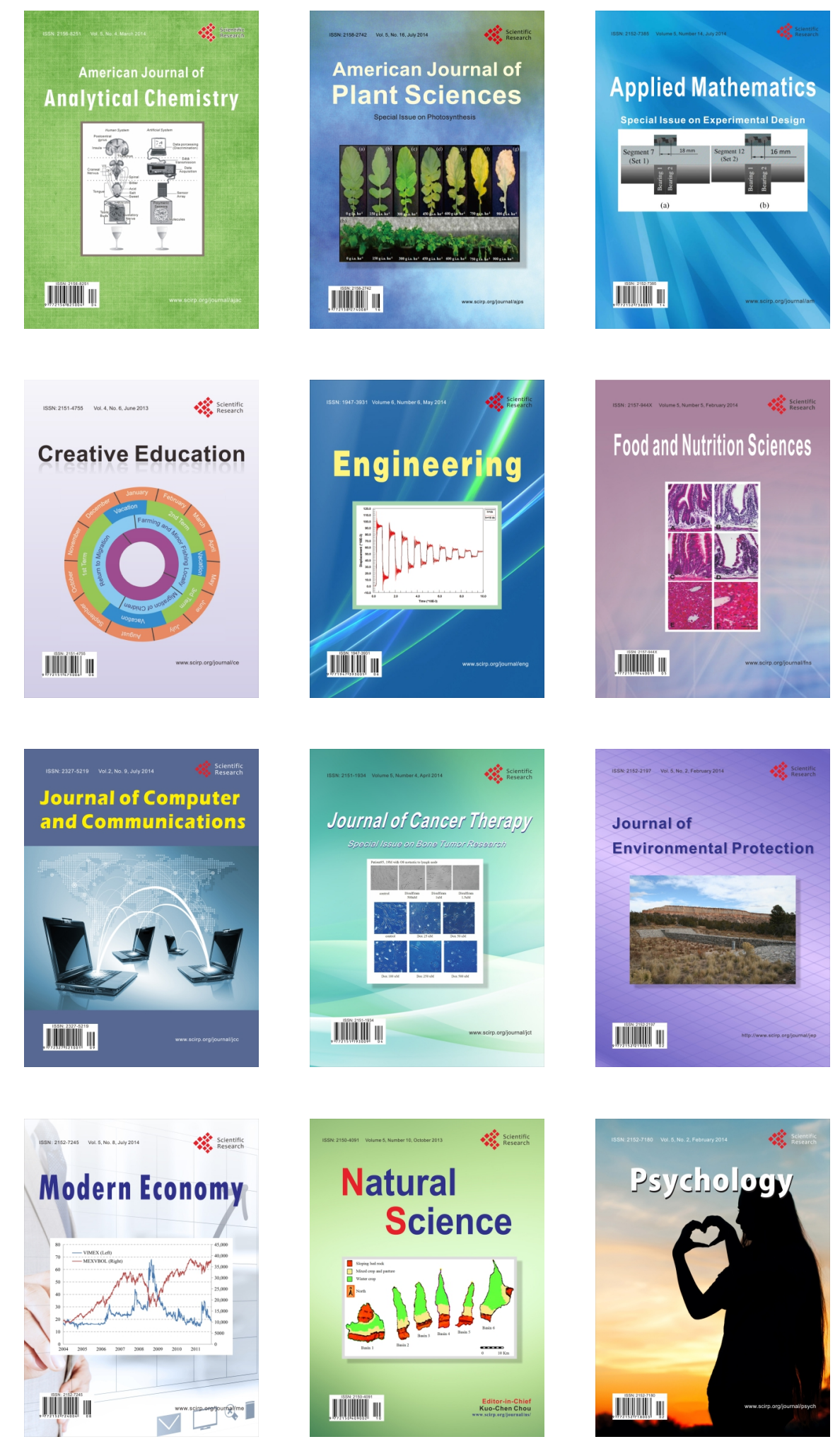